\title{
The Association of Metabolic Syndrome with Executive Dysfunction Independent of Subclinical Ischemic Brain Lesions in Japanese Adults
}

\author{
Hirokazu Bokura ${ }^{a}$ Atsushi Nagai ${ }^{b}$ Hiroaki Oguro ${ }^{a}$ Shotai Kobayashi ${ }^{c}$ \\ Shuhei Yamaguchi ${ }^{\mathrm{a}}$ \\ Departments of a Neurology and ${ }^{\mathrm{b}}$ Laboratory Medicine, Faculty of Medicine, Shimane University, and \\ 'Shimane University Hospital, Izumo, Japan
}

\section{Key Words}

Metabolic syndrome $\cdot$ Cognitive impairment .

Executive function $\cdot$ Brain lesions $\cdot$ Glucose metabolism

\begin{abstract}
Background/Aims: The aim of this study is to examine whether metabolic syndrome is directly associated with cognitive impairment independent of subclinical ischemic brain lesions. Methods: We examined 1,543 neurologically normal subjects (44-86 years of age). Metabolic syndrome, brain MRI, and cognitive functions including general cognition and executive function were assessed. Results: After adjusting for silent brain lesions and demographic risk factors, metabolic syndrome was an independent risk factor for impairment of executive function $(\mathrm{OR}=1.41,95 \% \mathrm{Cl}=1.17-1.25$ for Kohs' Test; $\mathrm{OR}=2.25,95 \% \mathrm{Cl}=1.64-3.07$ for Frontal Assessment Battery). Among the components of metabolic syndrome, elevated fasting glucose was the only independent risk factor for impairment of executive function. Conclusion: Metabolic syndrome is associated with impairment of executive function independent of silent brain lesions in the Japanese population.

Copyright $\odot 2011$ S. Karger AG, Basel
\end{abstract}

(C) 2011 S. Karger AG, Basel

$1420-8008 / 10 / 0306-0479 \$ 26.00 / 0$

Fax +4161306 1234

E-Mail karger@karger.ch

www.karger.com
Accessible online at:

www.karger.com/dem

\section{Introduction}

Metabolic syndrome has been recognized as a risk factor not only for cardiovascular disease but also for stroke $[1,2]$. Moreover, recent studies showed that metabolic syndrome was significantly associated with silent brain infarctions (SBI) [3, 4], periventricular hyperintensity $(\mathrm{PVH})$, and silent subcortical white matter lesions (SWML) $[4,5]$.

Of the components that make up metabolic syndrome, hypertension is thought to be a risk factor for dementia [6-8], and antihypertensive therapy has an impact on the prevalence of Alzheimer's disease $[9,10]$ and vascular dementia. Patients with diabetes mellitus were also reported to be at higher risk of developing dementia [11, 12]. Although the individual risk factors that make up metabolic syndrome each play some role in the decline of cognitive function, it is not entirely clear whether altogether these risk factors have a high predictive value for cognitive deterioration. A significant influence of metabolic syndrome on cognitive impairment in some domains has been demonstrated in recent cohort studies with either a cross-sectional $[13,14]$ or longitudinal research design [15]. Some population-based cohort study reported that Alzheimer's disease was more frequently detected in sub- 
jects with metabolic syndrome than in subjects without metabolic syndrome [16]. However, most cohort studies examining the association of metabolic syndrome and cognitive function have not assessed the contribution of subclinical ischemic brain lesions such as SBI, PVH or SWML, and some studies have included subjects with a history of stroke. Thus, it is unknown whether the association of metabolic syndrome with cognitive impairment depends on ischemic brain lesions. In the present cross-sectional study of neurologically normal middleaged and older subjects, we examined whether metabolic syndrome affects the incidence of cognitive impairment and whether these effects are dependent on SBI, PVH and SWML.

\section{Subjects and Methods}

\section{Subjects}

From November 2001 through March 2007, a total of 1,913 Japanese subjects voluntarily participated in the health checkup system in the Shimane Institute of Health Science. The checkup system included the collection of medical, neurological, and psychiatric history; formal neurological examinations by an experienced neurologist; neuropsychological testing; MRI scans of the head, and blood tests. We selected 1,543 neurologically normal subjects (44-86 years, mean age $62.0 \pm 6.2$ years) for this study from all participants. The criteria for subject exclusion were as follows: having any history of neurological or psychiatric diseases such as cerebrovascular diseases including transient ischemic attack, dementia, depression, or other psychiatric diseases $(n=364)$, and missing data to ensure complete analysis $(n=6)$. Demographic data are shown in table 1 . Compared to a population-based cohort study, this study had some biases in the background of the subjects in terms of their economical level, because only $17 \%$ of the participants were given financial supports by mutual aid associations and other subjects paid all expenses for the checkup by themselves. All subjects provided informed consent for this study, which was approved by the institutional ethics committee.

\section{Criteria for Metabolic Syndrome and Other Risk Factors}

Metabolic syndrome was diagnosed according to the modified criteria of the National Cholesterol Education Program Adult Treatment Panel III [17], adapted for the Japanese population [18]. Because waist circumference was not measured at the time of data acquisition, we used body mass index (BMI) as a substitute for waist circumference. There was a significant correlation between waist circumference and BMI in our previous study [19]. Based on the study, central obesity was defined as BMI $\geq 25$. In addition to central obesity, subjects with metabolic syndrome had to have 2 or more additional criteria, including elevated blood pressure or hypertension, elevated fasting glucose or diabetes mellitus, and/ or dyslipidemia. Blood pressure was measured 3 times after a 15min resting period, and the mean of 3 measurements was used in all further analysis. Elevated blood pressure was defined as a systolic blood pressure of $>130 \mathrm{~mm} \mathrm{Hg}$ and/or a diastolic blood pressure of $>85 \mathrm{~mm} \mathrm{Hg}$. Hypertension was defined as a systolic blood
Table 1. Demographic data and silent brain lesions

\begin{tabular}{lclc}
\hline & $\begin{array}{l}\text { Non-MetS } \\
(\mathrm{n}=1,357)\end{array}$ & $\begin{array}{l}\text { MetS } \\
(\mathrm{n}=186)\end{array}$ & $\mathrm{p}$ \\
\hline Gender (male/female) & $670 / 687$ & $144 / 42$ & $<0.0001$ \\
Age, years & $62.2 \pm 5.9$ & $61.2 \pm 7.9$ & n.s. \\
Education length, years & $12.4 \pm 2.5$ & $12.8 \pm 2.7$ & $<0.05$ \\
Smoking, \% & 39.9 & 62.1 & $<0.0001$ \\
Alcohol habit, \% & 14.8 & 30.6 & $<0.0001$ \\
Cardiovascular diseases, \% & 10.9 & 20.6 & $<0.005$ \\
SBI+, \% & 13.0 & 24.7 & $<0.0001$ \\
PVH+, \% & 3.8 & 5.9 & n.s. \\
SWML+, \% & 13.7 & 18.8 & n.s. \\
\hline
\end{tabular}

MetS = Metabolic syndrome; n.s. = not significant.

pressure $\geq 140 \mathrm{~mm} \mathrm{Hg}$, a diastolic blood pressure $\geq 90 \mathrm{~mm} \mathrm{Hg}$, and/or a history of hypertension with antihypertensive therapy based on the individual's medical history. Elevated fasting glucose was defined as a fasting glucose level $>110 \mathrm{mg} / \mathrm{dl}(6.1 \mathrm{mmol} / \mathrm{l})$. Diabetes mellitus was defined as a fasting serum glucose level $\geq 126 \mathrm{mg} / \mathrm{dl}$, a hemoglobin Alc level $\geq 6.5 \%$ or a medical history of diabetes mellitus. Dyslipidemia was defined as a plasma triglyceride level of $>150 \mathrm{mg} / \mathrm{dl}$ and/or a high-density lipoprotein cholesterol level of $<40 \mathrm{mg} / \mathrm{dl}$ or a medical history of dyslipidemia. A smoker was defined as any subject whose smoking index exceeded 200. Regular alcohol consumption was defined as more than $58 \mathrm{ml}$ of alcohol consumed per day. The incidence of cardiovascular diseases is described in table 1 .

\section{Imaging Data}

Head MRIs were performed using a 1.5-tesla MRI (Symphony Ultra Gradient, Siemens). The whole head was scanned with a $\mathrm{T}_{2^{-}}$

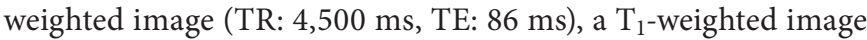
(TR: 2,500 ms, TE: $3.9 \mathrm{~ms}$ ), and fluid-attenuated inversion recovery images (TR: 8,000 ms, TE: $92 \mathrm{~ms}$ ) for the transverse plane, and $\mathrm{T}_{1}$-weighted image for the coronal plane, with a slice thickness of $7 \mathrm{~mm}$. Brain infarction was defined as a focal hyperintense lesion of $3 \mathrm{~mm}$ or larger in diameter in the $\mathrm{T}_{2}$-weighted image corresponding to a hypointense lesion in the $\mathrm{T}_{1}$-weighted image. $\mathrm{PVH}$ and SWML were evaluated separately based on their distinct subcortical distributions on the fluid-attenuated inversion recovery image, because PVH is observed adjacent to the ventricles and SWML is observed separate from the ventricles. PVH was graded on a scale of $0-4$ as described elsewhere [20]. SWML was graded on a scale of 0-3 according to Fazekas et al.'s grading scheme [21]. For statistical purposes, PVH and SWML grades were dichotomized; we defined PVH grades $0-2$ as 'PVH-' and grades $3-4$ as 'PVH+'; similarly, SWML grades $0-1$ were defined as 'SWML-', and grades 2-3 were termed 'SWML+'. All MRI findings were read and determined separately by an experienced neurologist and neuroradiologist who were blinded to the patients' profiles. When their opinions were inconsistent, a second neurologist was brought in for consultation. An interrater study for evaluating MRI lesions was also performed by 2 independent raters in a blinded fashion. The agreement rate was $94 \%$. 
Cognitive Functions

Okabe's Intelligence Scale (Okabe's Test) [22], which is a shortened and modified Wechsler Adult Intelligence Scale-Revised for the Japanese aged population, was used for assessing general intelligence, including orientation, semantic memory, calculation, forward and backward digit span, and paired association memory. The test is scored out of a total of 60 points and its reliability and validity had been reported [23]. There was a significant correlation between Okabe's Test and Mini-Mental Scale Examination $(\sigma$ value $=0.76, \mathrm{n}=426, \mathrm{p}<0.0001$ by Spearman's rank correlation analysis). Executive function was assessed using 2 tests. One was the Kohs Block Design Test (Kohs' test), in which subjects are shown cards with a variety of colored designs and asked to reproduce them using a set of colored blocks, yielding an intelligence quotient [24]. This test assessed the visuospatial ability in addition to executive function. Another test for executive function was the Frontal Assessment Battery (FAB), which is a composite tool consisting of 6 subtests, i.e. categorization, flexibility, programming, resistance to interference, inhibitory control, and forced movement $[25,26]$. We converted an individual value of the cognitive test score to a $\mathrm{Z}$-value on the basis of an institutional database of age-matched normal subjects.

\section{Statistical Analysis}

We used Student's t test for the group comparison of parametric variables. The Mann-Whitney $U$ test and $\chi^{2}$ test were used for nonparametric statistics. Since the distributions of cognitive function test scores did not show normal distribution, they were treated as ordinal variables. Univariate and multivariate ordered logistic regression models were used to determine the association between cognitive function and metabolic syndrome with MRI changes and demographic risk factors as independent variables. We also examined the association between cognitive function and each component of metabolic syndrome with ordered logistic regression analysis with adjustment for MRI changes and demographic risk factors. All statistical analyses were performed using IBM SPSS Statistics ver. 19 (SPSS, Inc.). Differences with $\mathrm{p}<0.05$ were considered significant.

\section{Results}

Characteristics and Prevalence of Silent Brain Lesions

Of the 1,543 subjects, 186 (12.1\%) had metabolic syndrome. The clinical characteristics and the prevalence of silent brain lesions are presented in table 1 . There was no difference in age between the metabolic syndrome and non-metabolic syndrome groups. There were more male subjects with more education in the metabolic syndrome group. Moreover, the metabolic syndrome group had higher rates of smoking, alcohol use and incidence of cardiovascular diseases than the non-metabolic syndrome group. The prevalence of SBI was $13.0 \%$ in the non-metabolic syndrome group and $24.7 \%$ in the metabolic syndrome group $(\mathrm{p}<0.0001)$, whereas there were no significant differences in the prevalence of $\mathrm{PVH}+$ and SWML+ between the metabolic syndrome and non-metabolic syndrome groups.

\section{Cognitive Function and Metabolic Syndrome}

Unadjusted univariate regression models indicated that decreases of Kohs' Test and FAB scores were significantly associated with metabolic syndrome $(\mathrm{OR}=1.34$, $95 \% \mathrm{CI}=1.03-1.73, \mathrm{p}=0.028$ for Kohs' Test, and OR = $2.28,95 \% \mathrm{CI}=1.66-3.09, \mathrm{p}<0.0001$ for $\mathrm{FAB}$ ), whereas the association with Okabe's Test was marginal (OR = $1.29,95 \% \mathrm{CI}=0.99-1.66, \mathrm{p}=0.056)$. As there were significant associations between cognitive function test scores and demographic data (i.e. age, gender, and education level), we performed multivariate ordered logistic regression analysis using metabolic syndrome as an independent variable and all cognitive function test scores as dependent variables with adjustment for age, gender, and education level. This analysis demonstrated significant associations of metabolic syndrome with decreases in Kohs' Test and FAB scores but not with decreases in Okabe's Test score (model 1 in table 2). Furthermore, we examined a model by adding interactions of metabolic syndrome and demographic data as variables, because the interaction might modify the associations. However, cognitive function scores were not modified by the interactions.

Then, we performed ordered logistic regression analysis including silent brain lesions as independent variables with adjustments for demographic factors. As seen in model 2 in table 2, metabolic syndrome was a risk factor for decreases in Kohs' Test and FAB scores independent of age, gender, education level, and silent brain lesions. Since silent brain lesions may affect cognitive impairment interactively with metabolic syndrome, we examined the effect of interaction of metabolic syndrome and MRI changes on cognitive functions. The analysis revealed no significant effects of any interaction terms on cognitive functions.

Furthermore, another model including other demographic risk factors (smoking and alcohol) was examined. The analysis demonstrated that significant associations of metabolic syndrome with decreases in Kohs' Test and FAB scores were still observed after adjustment for smoking and alcohol (model 3 in table 2).

Finally, we examined the association between impairment of cognitive functions and each component that makes up metabolic syndrome. After adjusting for all demographical risk factors and silent brain lesions, multivariate ordered logistic regression analyses demonstrated that of all components for metabolic syn- 
Table 2. Association of metabolic syndrome and other demographic factors with cognitive impairment

\begin{tabular}{|c|c|c|c|c|c|c|}
\hline Variables & OR & $\mathrm{p}$ & OR & $\mathrm{p}$ & OR & $\mathrm{p}$ \\
\hline \multicolumn{7}{|l|}{ Model 1} \\
\hline MetS & $1.25(0.96-1.62)$ & 0.10 & $1.48(1.14-1.92)$ & 0.003 & $2.25(1.65-3.07)$ & $<0.0001$ \\
\hline \multicolumn{7}{|l|}{ Model 2} \\
\hline MetS & $1.21(0.93-1.57)$ & 0.15 & $1.45(1.11-1.89)$ & 0.005 & $2.24(1.64-3.06)$ & $<0.0001$ \\
\hline SWML+ & $1.01(0.78-1.30)$ & 0.96 & $1.35(1.04-1.75)$ & 0.02 & $1.11(0.82-1.52)$ & 0.50 \\
\hline \multicolumn{7}{|l|}{ Model 3} \\
\hline MetS & $1.20(0.92-1.57)$ & 0.17 & $1.41(1.17-1.25)$ & 0.01 & $2.25(1.64-3.07)$ & $<0.0001$ \\
\hline SBI+ & $1.25(0.97-1.62)$ & 0.08 & $1.02(0.79-1.31)$ & 0.91 & $1.29(0.96-1.74)$ & 0.09 \\
\hline PVH+ & $1.09(0.70-1.71)$ & 0.70 & $1.26(0.80-1.98)$ & 0.31 & $0.80(0.48-1.32)$ & 0.38 \\
\hline
\end{tabular}

All 3 models were adjusted for age, gender, and education length. MetS = Metabolic syndrome.

Figures in parentheses indicate $95 \%$ CIs.

Table 3. Association of components of metabolic syndrome with cognitive impairment

\begin{tabular}{|c|c|c|c|c|}
\hline & \multicolumn{2}{|c|}{ Decrease in Kohs' test scores } & \multicolumn{2}{|c|}{ Decrease in FAB scores } \\
\hline & OR & $\mathrm{p}$ & OR & $\mathrm{p}$ \\
\hline Increased BMI & $1.33(0.97-1.79)$ & 0.06 & $1.18(0.90-1.68)$ & 0.30 \\
\hline Elevated blood pressure & $1.25(0.95-1.68)$ & 0.10 & $1.05(0.85-1.38)$ & 0.55 \\
\hline Dyslipidemia & $0.81(0.56-1.10)$ & 0.18 & $1.18(0.90-1.58)$ & 0.20 \\
\hline Elevated fasting glucose & $1.52(1.07-1.98)$ & 0.01 & $1.41(1.05-1.88)$ & 0.02 \\
\hline
\end{tabular}

The analysis was adjusted for age, gender, education length, smoking, alcohol habit, and silent brain lesions.

Figures in parentheses indicate 95\% CIs. drome only elevated fasting glucose was an independent risk factor for decreases in Kohs' Test and FAB scores (table 3).

\section{Discussion}

Recent studies have demonstrated that metabolic syndrome is a risk factor for cognitive impairment, and it is associated with an increased risk of developing cognitive impairment related to Alzheimer's disease and vascular dementia $[16,27,28]$. However, it was not clear whether this cognitive impairment is associated with structural changes in the brain or whether the association between metabolic syndrome and cognitive impairment is observed even in healthy subjects. In this study, we found that metabolic syndrome was associated with cognitive impairment in executive function independent of silent brain lesions in healthy subjects.

Kwon et al. [3] reported that metabolic syndrome was an independent risk factor for SBI in healthy people. In the same way, Park et al. [5] found that metabolic syndrome was associated with leukoaraiosis. In addition, we recently found that metabolic syndrome was a risk factor for silent brain lesions, including SBI, severe PVH and SWML [4]. Furthermore, elderly people with SBI have 
been reported to have a high risk of dementia and cognitive impairment [29]. Several reports have shown that white matter hyperintensity on MRI was similarly associated with cognitive impairment and depression [30-33]. These findings suggest that ischemic brain damage may contribute to the association between metabolic syndrome and cognitive impairment. However, we found that metabolic syndrome was associated with cognitive impairment independent of ischemic brain lesions, indicating that cognitive impairment facilitated by metabolic syndrome may be linked to a neurodegenerative etiology rather than vascular etiology.

There are several studies suggesting an association between metabolic syndrome and the degenerative disorder of dementia. Yaffe et al. [34] prospectively studied the influence of metabolic syndrome on a 4-year risk of developing cognitive impairment (dementia or mild cognitive impairment) in 4,895 older women. In their study, 497 women $(10.2 \%)$ had metabolic syndrome, and of those women, 36 (7.2\%) developed cognitive impairment (ageadjusted $\mathrm{OR}=1.7,95 \% \mathrm{CI}=1.1-2.4$ ). Razay et al. [35] also reported a case-control study of 50 consecutive patients in which metabolic syndrome was associated with Alzheimer's disease $(\mathrm{OR}=3.2,95 \% \mathrm{CI}=1.2-8.4)$. In their study, patients with Alzheimer's disease had lower mean systolic blood pressure, and the association between metabolic syndrome and Alzheimer's disease was strengthened when hypertension was excluded as a factor $(\mathrm{OR}=$ 7.0, 95\% CI $=2.7-18.3$ ). Vanhanen et al. [16] also reported an association between metabolic syndrome and Alzheimer's disease. They showed in a population-based study that Alzheimer's disease was more frequently detected in subjects with metabolic syndrome than in those without it. Although these studies confirmed the association of metabolic syndrome with Alzheimer's disease, there has been no evidence that metabolic syndrome contributes to the early impairment of cognitive function in healthy subjects. The present study demonstrated that metabolic syndrome affects cognitive function even in healthy middle-aged and older subjects.

A previous study suggests that the atherosclerotic process may underlie the association between metabolic syndrome and cognitive impairment, based on higher values of inflammation markers [36, 37]. In the present study, we adjusted for subclinical ischemic changes of the brain, which suggests that another mechanism besides atherosclerosis may account for the association. Inflammation is reported to contribute to degenerative processes in both Alzheimer's disease and metabolic syndrome [38, 39]. Of all the risk factors that comprise metabolic syn- drome, hyperglycemia is reported to produce toxic hyperglycosylated end products, leading to lowered amyloid degradation $[40,41]$. The employment of precise neuroimaging for examining anatomical or metabolic changes in the brain might clarify the effect of metabolic syndrome on the neural degeneration process.

Metabolic syndrome affected executive functions. This finding is consistent with a recent case-control study, in which executive functions and speed of mental processing were selectively impaired in subjects with metabolic syndrome [14]. The authors speculated smallvessel diseases as an underlying pathological basis for executive dysfunction. However, this hypothesis is not entirely supported by the present study, because the association of metabolic syndrome and executive dysfunction was independent of MRI changes related to small-vessel diseases (i.e. SBI, PVH, and SWML). Rather, hyperglycemia or glucose intolerance might have some role in executive dysfunction since elevated fasting glucose was an independent and higher risk factor for impairment in executive function in the present study. This finding is consistent with a recent report in which hyperglycemia was a main contributor to the association of metabolic syndrome with executive function [42]. The cognitive functions that are performed in frontal-subcortical brain structures were predominantly affected by diabetes and glucose intolerance [43]. On the other hand, elevated blood pressure was not an independent risk factor for cognitive impairment in the present study, although there is abundant evidence that hypertension is a strong risk factor for cognitive impairment $[44,45]$. Because hypertension is a strong risk factor for ischemic brain insults, the association of hypertension with cognitive impairment may have been obscured when silent brain lesions were adjusted for in the multivariate analysis.

The present study has several limitations. Because we lacked the data of waist circumference, which reflects central obesity more accurately, BMI was used in this study as a substitute of waist circumference after the confirmation of good correlation between these 2 measures. Furthermore, since the Japanese criteria of metabolic syndrome are different from those of other countries, the present findings may not be relevant to other populations. A causal role for metabolic syndrome on cognitive impairment is indistinct because of the cross-sectional data. A longitudinal study is necessary to address whether cognitive impairment is associated with the future development of dementia. There is a selection bias in that subjects who were concerned about changes in their cog- 
nitive function and silent brain lesions may have participated in this volunteer-based cohort study. There is also a bias in the background of participants compared to a population-based cohort study in terms of their economical level as described in the Subjects and Methods section. Thus, some caution may be necessary to generalize the present finding to other populations. Despite these limitations, we obtained both brain imaging data and cognitive function data at the same time in a fairly large number of middle-aged and older healthy persons. This enabled us to address the issue whether the association of metabolic syndrome and cognitive function is independent of ischemic silent brain lesions.

\section{Acknowledgments}

This research was supported by the Shimane Institute of Health Science (2003-2006) and Mitsubishi Pharma Research Foundation (2009).

\section{References}

1 Lakka HM, Laaksonen DE, Lakka TA, Niskanen LK, Kumpusalo E, Tuomilehto J, Salonen JT: The metabolic syndrome and total and cardiovascular disease mortality in middle-aged men. JAMA 2002;288:2709-2716.

-2 Kurl S, Laukkanen JA, Niskanen L, Laaksonen D, Sivenius J, Nyyssonen K, Salonen JT: Metabolic syndrome and the risk of stroke in middle-aged men. Stroke 2006;37: 806-811.

-3 Kwon HM, Kim BJ, Lee SH, Choi SH, Oh BH, Yoon BW: Metabolic syndrome as an independent risk factor of silent brain infarction in healthy people. Stroke 2006;37:466-470.

4 Bokura H, Yamaguchi S, Iijima K, Nagai A, Oguro H: Metabolic syndrome is associated with silent ischemic brain lesions. Stroke 2008;39:1607-1609.

5 Park K, Yasuda N, Toyonaga S, Yamada SM, Nakabayashi H, Nakasato M, Nakagomi T, Tsubosaki E, Shimizu K: Significant association between leukoaraiosis and metabolic syndrome in healthy subjects. Neurology 2007;69:974-978

-6 Vicario A, Martinez CD, Baretto D, Diaz Casale A, Nicolosi L: Hypertension and cognitive decline: impact on executive function. J Clin Hypertens (Greenwich) 2005;7:598604.

7 Qiu C, Winblad B, Fratiglioni L: The age-dependent relation of blood pressure to cognitive function and dementia. Lancet Neurol 2005;4:487-499.

8 Skoog I, Gustafson D: Update on hypertension and Alzheimer's disease. Neurol Res 2006;28:605-611.

-9 Kalmijn S, Foley D, White L, Burchfiel CM, Curb JD, Petrovitch H, Ross GW, Havlik RJ, Launer LJ: Metabolic cardiovascular syndrome and risk of dementia in JapaneseAmerican elderly men. The Honolulu-Asia Aging Study. Arterioscler Thromb Vasc Biol 2000;20:2255-2260.

10 Staessen JA, Wang JG, Thijs L: Calciumchannel blockade and cardiovascular prognosis: recent evidence from clinical outcome trials. Am J Hypertens 2002;15:85S-93S.
11 MacKnight C, Rockwood K, Awalt E, McDowell I: Diabetes mellitus and the risk of dementia, Alzheimer's disease and vascular cognitive impairment in the Canadian study of health and aging. Dement Geriatr Cogn Disord 2002;14:77-83.

12 Ott A, Stolk RP, van Harskamp F, Pols HA, Hofman A, Breteler MM: Diabetes mellitus and the risk of dementia: the Rotterdam Study. Neurology 1999;53:1937-1942.

13 Hassenstab JJ, Sweat V, Bruehl H, Convit A: Metabolic syndrome is associated with learning and recall impairment in middle age. Dement Geriatr Cogn Disord 2010;29: 356-362.

14 Segura B, Jurado MA, Freixenet N, Albuin C, Muniesa J, Junque C: Mental slowness and executive dysfunctions in patients with metabolic syndrome. Neurosci Lett 2009;462: 49-53.

15 Akbaraly TN, Kivimaki M, Shipley MJ, Tabak AG, Jokela M, Virtanen M, Marmot MG, Ferrie JE, Singh-Manoux A: Metabolic syndrome over 10 years and cognitive functioning in late midlife: the Whitehall II Study. Diabetes Care 2010;33:84-89.

16 Vanhanen M, Koivisto K, Moilanen L, Helkala EL, Hanninen T, Soininen $H$, Kervinen K, Kesaniemi YA, Laakso M, Kuusisto J: Association of metabolic syndrome with Alzheimer disease: a population-based study. Neurology 2006;67:843-847.

$\checkmark 17$ Executive summary of the third report of the National Cholesterol Education Program (NCEP) expert panel on detection, evaluation, and treatment of high blood cholesterol in adults (Adult Treatment Panel III). JAMA 2001;285:2486-2497.

18 Matsuzawa Y: Metabolic syndrome - definition and diagnostic criteria in Japan. J Atheroscler Thromb 2005;12:301.

19 Takahashi K, Bokura H, Kobayashi S, Iijima K, Nagai A, Yamaguchi S: Metabolic syndrome increases the risk of ischemic stroke in women. Intern Med 2007;46:643-648.
20 Kobayashi S, Okada K, Koide H, Bokura H, Yamaguchi S: Subcortical silent brain infarction as a risk factor for clinical stroke. Stroke 1997;28:1932-1939.

21 Fazekas F, Niederkorn K, Schmidt R, Offenbacher H, Horner S, Bertha G, Lechner H: White matter signal abnormalities in normal individuals: correlation with carotid ultrasonography, cerebral blood flow measurements, and cerebrovascular risk factors. Stroke 1988;19:1285-1288.

22 Fukunishi I, Okabe S, Nakagawa T, Hosokawa $\mathrm{K}$ : The assessment of intelligence function of aged chronic schizophrenia. Jpn J Psychiatry Neurol 1990;44:503-509.

23 Okabe S: Simple intelligence assessment scale. Jpn J Clin Psychiat 1988;17:55-61.

24 Wigg CM, Duro LA: The Kohs' blocks test as an important instrument to investigate the visuo-spatial impairments in myotonic dystrophy. 1. Quantitative and qualitative analysis. Arq Neuropsiquiatr 1999;57:547-555.

25 Dubois B, Slachevsky A, Litvan I, Pillon B: The FAB: a Frontal Assessment Battery at bedside. Neurology 2000;55:1621-1626.

26 Mok VC, Wong A, Yim P, Fu M, Lam WW, Hui AC, Yau C, Wong KS: The validity and reliability of Chinese frontal assessment battery in evaluating executive dysfunction among Chinese patients with small subcortical infarct. Alzheimer Dis Assoc Disord 2004; $18: 68-74$

27 Muller M, Tang MX, Schupf N, Manly JJ, Mayeux R, Luchsinger JA: Metabolic syndrome and dementia risk in a multiethnic elderly cohort. Dement Geriatr Cogn Disord 2007;24:185-192.

-28 Yaffe K, Haan M, Blackwell T, Cherkasova E, Whitmer RA, West N: Metabolic syndrome and cognitive decline in elderly Latinos: findings from the Sacramento Area Latino Study of Aging study. J Am Geriatr Soc 2007; 55:758-762.

-29 Vermeer SE, Den Heijer T, Koudstaal PJ, Oudkerk M, Hofman A, Breteler MM: Incidence and risk factors of silent brain infarcts in the population-based Rotterdam Scan Study. Stroke 2003;34:392-396. 
30 Roman GC, Erkinjuntti T, Wallin A, Pantoni L, Chui HC: Subcortical ischaemic vascular dementia. Lancet Neurol 2002;1:426-436.

-31 Price CC, Jefferson AL, Merino JG, Heilman KM, Libon DJ: Subcortical vascular dementia: integrating neuropsychological and neuroradiologic data. Neurology 2005;65:376382.

-32 Ylikoski R, Jokinen H, Andersen P, Salonen O, Madureira S, Ferro J, Barkhof F, van der Flier W, Schmidt R, Fazekas F, Scheltens P, Waldemar G, Salvadori E, Pantoni L, Inzitari D, Erkinjuntti T: Comparison of the Alzheimer's disEase Assessment Scale Cognitive Subscale and the Vascular Dementia Assessment Scale in differentiating elderly individuals with different degrees of white matter changes. The LADIS study. Dement Geriatr Cogn Disord 2007;24:73-81.

-33 Teodorczuk A, O’Brien JT, Firbank MJ, Pantoni L, Poggesi A, Erkinjuntti T, Wallin A, Wahlund LO, Gouw A, Waldemar G, Schmidt R, Ferro JM, Chabriat H, Bazner H, Inzitari $\mathrm{D}$ : White matter changes and latelife depressive symptoms: longitudinal study. Br J Psychiatry 2007;191:212-217.
34 Yaffe K, Weston AL, Blackwell T, Krueger KA: The metabolic syndrome and development of cognitive impairment among older women. Arch Neurol 2009;66:324-328.

35 Razay G, Vreugdenhil A, Wilcock G: The metabolic syndrome and Alzheimer disease. Arch Neurol 2007;64:93-96.

36 Dik MG, Jonker C, Comijs HC, Deeg DJ, Kok A, Yaffe K, Penninx BW: Contribution of metabolic syndrome components to cognition in older individuals. Diabetes Care 2007;30:2655-2660.

37 Milionis HJ, Rizos E, Goudevenos J, Seferiadis K, Mikhailidis DP, Elisaf MS: Components of the metabolic syndrome and risk for first-ever acute ischemic nonembolic stroke in elderly subjects. Stroke 2005;36:13721376.

38 Erol A: An integrated and unifying hypothesis for the metabolic basis of sporadic Alzheimer's disease. J Alzheimers Dis 2008; 13: 241-253.

39 Watson GS, Craft S: Insulin resistance, inflammation, and cognition in Alzheimer's disease: lessons for multiple sclerosis. J Neurol Sci 2006;245:21-33.

40 Biessels GJ, Kappelle LJ: Increased risk of Alzheimer's disease in type II diabetes: insulin resistance of the brain or insulin-induced amyloid pathology? Biochem Soc Trans 2005;33:1041-1044.
41 Xie L, Helmerhorst E, Taddei K, Plewright B, Van Bronswijk W, Martins R: Alzheimer's beta-amyloid peptides compete for insulin binding to the insulin receptor. J Neurosci 2002;22:RC221.

42 Schuur M, Henneman P, van Swieten JC, Zillikens MC, de Koning I, Janssens AC, Witteman JC, Aulchenko YS, Frants RR, Oostra $\mathrm{BA}$, van Dijk KW, van Duijn CM: Insulinresistance and metabolic syndrome are related to executive function in women in a large family-based study. Eur J Epidemiol 2010;25:561-568.

43 Geroldi C, Frisoni GB, Paolisso G, Bandinelli S, Lamponi M, Abbatecola AM, Zanetti O, Guralnik JM, Ferrucci L: Insulin resistance in cognitive impairment: the Inchianti Study. Arch Neurol 2005;62:1067-1072.

44 Launer LJ, Masaki K, Petrovitch H, Foley D, Havlik RJ: The association between midlife blood pressure levels and late-life cognitive function. The Honolulu-Asia Aging Study. JAMA 1995;274:1846-1851.

45 Knopman D, Boland LL, Mosley T, Howard G, Liao D, Szklo M, McGovern P, Folsom AR: Cardiovascular risk factors and cognitive decline in middle-aged adults. Neurology 2001;56:42-48. 